\title{
Randomly Weighted Averages on Order Statistics
}

\author{
Homei Hajir, Hasanzadeh Leila, Mina Ghasemi \\ Department of Statistics, Faculty of Mathematical Sciences, University of Tabriz, Tabriz, Iran \\ Email: homei@tabrizu.ac.ir.
}

Received July 23, 2012; revised January 4, 2013; accepted January 11, 2013

Copyright (C) 2013 Homei Hajir et al. This is an open access article distributed under the Creative Commons Attribution License, which permits unrestricted use, distribution, and reproduction in any medium, provided the original work is properly cited.

\begin{abstract}
We study a well-known problem concerning a random variable uniformly distributed between two independent random variables. Two different extensions, randomly weighted average on independent random variables and randomly weighted average on order statistics, have been introduced for this problem. For the second method, two-sided power random variables have been defined. By using classic method and power technical method, we study some properties for these random variables.
\end{abstract}

Keywords: Two-Sided Power; Moment; Weighted Averages; Power Distribution

\section{Introduction}

Van Asch [1] introduced the notion of a random variable $Z$ uniformly distributed between two independent random variables $X_{1}$ and $X_{2}$ which arose in studying the distribution of products of random $2 \times 2$ matrices for stochastic search of global maxima. By letting $X_{1}$ and $X_{2}$ to have identical distribution, he derived that: 1) for $X_{1}$ and $X_{2}$ on $[-1,1], Z$ is uniform on $[-1,1]$ if and only if $X_{1}$ and $X_{2}$ have an Arcsine distribution; and 2) $Z$ possesses the same distribution as $X_{1}$ and $X_{2}$ if and only if $X_{1}$ and $X_{2}$ are degenerated or have a Cauchy distribution. Soltani and Homei [2] following Johnson and Kotz [3] extended Van Asch's results. They put $X_{1}, \cdots, X_{n}$ to be independent, and considered

$$
S_{n}=R_{1} X_{1}+R_{2} X_{2}+\cdots+R_{n-1} X_{n-1}+R_{n} X_{n}, n \geq 2 .
$$

where random proportions

$$
\begin{gathered}
R_{i}=U_{(i)}-U_{(i-1)}, i=1, \cdots, n-1, \\
R_{n}=1-\sum_{i=0}^{n-1} R_{i}, U_{(1)}, \cdots, U_{(n)}
\end{gathered}
$$

are order statistics from a uniform distribution on $[0,1]$, and $U_{(0)}=0$. These random proportions are uniformly distributed over the unit simplex. They employed Stieltjes transform and that: 1) $S_{n}$ possesses the same distribution as $X_{1}, \cdots, X_{n}$ if and only if $X_{1}, \cdots, X_{n}$ are degenerated or have a Cauchy distribution; and 2) Van Asch's result for Arcsine holds for $Z$ only.

In this paper, we introduce two families of distributions, suggested by an anonymous referee of the article, to whom the author expresses his deepest gratitude. We say that $Z_{1}$ is a random variable between two independent random variables with power distribution, if the conditionally distribution of $Z_{1}$ given at $X_{1}=x_{1}, X_{2}=x_{2}$ is

$$
F_{Z_{1} \mid x_{1}, x_{2}(z)}= \begin{cases}1 & z \geq \max \left(x_{1}, x_{2}\right), \\ \left(\frac{z-x_{1}}{x_{2}-x_{1}}\right)^{n} & x_{1}<z<x_{2}, \\ 1-\left(\frac{z-x_{1}}{x_{2}-x_{1}}\right)^{n} & x_{2}<z<x_{1}, \\ 1 & z \geq \max \left(x_{1}, x_{2}\right) .\end{cases}
$$

The distribution $F_{Z_{1} \mid x_{1}, x_{2}}(z)$ will be said to follow a conditionally directed power distribution, When $n$ is an integer. For $n=1$, the distribution given by (1.1) simplifies to the distribution $Z$ that was introduced before. Also we used Stieltjes methods, for more on the Stieltjes transform, see Zayed [4].

For $n=2$, we call $Z_{1}$ directed triangular random variable. For further generalizing Van Asch results, we introduce a seemingly more natural conditionally power distribution. We call $Z_{2}$ two-sided power (TSP) random variable if the conditionally distribution of $Z_{2}$ given at $X_{1}=x_{1}, X_{2}=x_{2}$ is

$$
F_{Z_{2} \mid x_{1}, x_{2}(z)}= \begin{cases}1 & z \geq y_{2}, \\ \left(\frac{z-y_{1}}{y_{2}-y_{1}}\right)^{n} & y_{1}<z<y_{2} . \\ 0 & z \leq y_{1} .\end{cases}
$$


The distribution $F_{Z_{2} \mid x_{1}, x_{2}}$ will be said to follow a conditionally undirected power distribution, when $y_{1}=\min$ $y_{1}=\min \left(x_{1}, x_{2}\right), y_{2}=\max \left(x_{1}, x_{2}\right)$ and $n$ is an integer.

Again for $n=1$, the distribution given by (1.1) simplifies to the distribution $Z$ that was introduced by Van Asch. The main aim of this article is providing a generalization of notion to the results of Van Asch for some other values of $n$ (other than $n=1$ ). This article is organized as follows. We introduce preliminaries and previous works in Section 2. In Section 3, we give some Characterizations for distribution of $Z_{1}$ given in (1.1), when $n=2$. In Section 4, we find distribution of $Z_{2}$ given in (1.2) by direct and power method, and give some examples and Characterizations of such distributions by use of Soltani and Homei' results [5].

\section{Preliminaries and Previous Works}

In this section, we first review some results of Van Asch [1] and then modify them a little Bit to fit in our framework, to be introduced in the forthcoming sections. Using the Heaviside function $(U(x)=0, x<0, x=1, x \geq 0)$, we conclude that for any given distinct values $X_{1}$ and $X_{2}$ the conditional distribution $F_{Z_{1} \mid x_{1}, x_{2}}(z)$ in (1.1) is

$$
\begin{aligned}
F_{Z_{1} \mid x_{1}, x_{2}}(z)= & \left(\frac{z-x_{1}}{x_{2}-x_{1}}\right)^{n} U\left(z-x_{1}\right) \\
& -\sum_{i=1}^{n}\left(\begin{array}{c}
n \\
i
\end{array}\right)\left(\frac{z-x_{1}}{x_{2}-x_{1}}\right)^{i} U\left(z-x_{2}\right) .
\end{aligned}
$$

Lemma 2.1. For distinct real's $x_{1}, x_{2}, z$ and integer $n$, we have

$$
\begin{aligned}
& \frac{-1}{\left(z-x_{1}\right)\left(x_{2}-x_{1}\right)}+\frac{(-1)^{n}}{(n-1) !} \frac{\mathrm{d}^{n-1}}{\mathrm{~d} x_{2}^{n-1}}\left(\frac{1}{\left(z-x_{2}\right)} \cdot \frac{1}{\left(x_{2}-x_{1}\right)}\right) \\
& =\frac{1}{\left(x_{1}-z\right)\left(x_{2}-z\right)^{n}} .
\end{aligned}
$$

Proof. It follows from the Leibniz formula.

Let $h \in D^{\alpha}(I)$, where $I \subseteq \mathbb{R}$ is an interval, and $D^{\alpha}(I)$ is the set of all real functions $f$ that are $\alpha$ Times differentiable on $I$. If $g(z)=\frac{1}{(z-x)}$. for some constants c and $k \in\{1, \cdots, n\}$. Then

$$
\begin{aligned}
P & \left.=M-g(z) \frac{\mathrm{d}^{k}}{\mathrm{dz}^{k}} h(z)+k g(z) \frac{\mathrm{d}^{k-1}}{\mathrm{dz}^{k-1}}\{h(z) g(z)\}-P\right] \\
& =\frac{\mathrm{d}^{k}}{\mathrm{~d} z^{k}}\{h(z) g(z)\} .
\end{aligned}
$$

We use the Leibnitz formula for the $(k-1)$ th derivative of a product, namely

$$
\begin{aligned}
& \frac{\mathrm{d}^{k-1}}{\mathrm{~d} z^{k-1}}\{h(z) g(z)\} \\
& =N=\sum_{i=0}^{k-1}\left(\begin{array}{c}
k-1 \\
i
\end{array}\right)\left\{\frac{\mathrm{d}^{i}}{\mathrm{~d} z^{i}} h(z)\right\}\left\{\frac{\mathrm{d}^{k-i}}{\mathrm{~d} z^{k-i}} g(z)\right\} .
\end{aligned}
$$

Let

$$
\frac{\mathrm{d}^{k}}{\mathrm{~d} z^{k}}\{h(z) g(z)\}=\frac{\mathrm{d}}{\mathrm{d} z} \frac{\mathrm{d}^{k-1}}{\mathrm{dz} z^{k-1}}\{h(z) g(z)\}=M+N .
$$

where

$$
\begin{gathered}
M=\sum_{i=0}^{k-1}\left(\begin{array}{c}
k-1 \\
i
\end{array}\right)\left\{\frac{\mathrm{d}^{i+1}}{\mathrm{~d} z^{i+1}} h(z)\right\}\left\{\frac{\mathrm{d}^{k-1-i}}{\mathrm{~d} z^{k-1-i}} g(z)\right\}, \\
N=\sum_{i=0}^{k-1}\left(\begin{array}{c}
k-1 \\
i
\end{array}\right)\left\{\frac{\mathrm{d}^{i}}{\mathrm{~d} z^{i}} h(z)\right\}\left\{\frac{\mathrm{d}^{k-i}}{\mathrm{~d}^{k-i}} g(z)\right\} .
\end{gathered}
$$

Since $\frac{\mathrm{d}^{r}}{\mathrm{~d} z^{r}} g(z)=\frac{r !}{(z-x)^{r+1}}$. It follows that

$\frac{\mathrm{d}^{r}}{\mathrm{~d} z^{r}} g(z)=r g(z)\left\{\frac{\mathrm{d}^{r-1}}{\mathrm{~d} z^{r-1}} g(z)\right\}$. Consequently, $N=k g(z) \frac{\mathrm{d}^{k-1}}{\mathrm{~d} z^{k-1}}\{h(z) g(z)\}-P$ where after some algebraic work $P=M-g(z) \frac{\mathrm{d}^{k}}{\mathrm{dz}^{k}} h(z)$.

Therefore,

$$
M+N=g(z)\left[\frac{\mathrm{d}^{k}}{\mathrm{~d} z^{k}} h(z)+k \frac{\mathrm{d}^{k-1}}{\mathrm{~d} z^{k-1}}\{h(z) g(z)\}\right] .
$$

This completes the proof.

Another tool for proving our main theorem is the following formula taken from the Schwartz Distribution theory, namely,

$$
\int_{-\infty}^{\infty} \varphi(x) \Lambda^{[n]}(\mathrm{d} x)=\frac{-1^{n}}{n !} \int_{-\infty}^{\infty} \frac{\mathrm{d}^{n}}{\mathrm{~d} x^{n}} \varphi(x) \Lambda(x)
$$

where $\Lambda$ is a distribution Function and $\Lambda^{[n]}$ is the $n$-th distributional derivative of $\Lambda$.

The conditional distribution $F_{Z_{1} \mid x_{1}, x_{2}}(z)$ given by (1.1) leads us to a linear functional on complex Valued functions $f: \mathbb{R} \rightarrow \mathbb{C}$, defined on the set of real numbers $\mathbb{R}$ :

$$
\begin{aligned}
& F_{Z_{1} \mid x_{1}, x_{2}}(f) \\
& =\frac{f\left(x_{1}\right)}{\left(x_{2}-x_{1}\right)^{n}}-\sum_{i=1}^{n} \frac{1}{(n-i) !\left(x_{2}-x_{1}\right)^{i}} \frac{\mathrm{d}^{n-i}}{\mathrm{dz}^{n-i}} f\left(x_{2}\right) .
\end{aligned}
$$

It easily follows that

$$
F_{Z_{1} \mid x_{1}, x_{2}}(a f+b g)=a F_{Z_{1} \mid x_{1}, x_{2}}(f)+b F_{Z_{1} \mid x_{1}, x_{2}}(g) .
$$

For any complex-valued functions $f, g$ and complex constants $a, b$. We note that $F_{Z_{1} \mid x_{1}, x_{2}}(z)=F_{Z_{1} \mid x_{1}, x_{2}}\left(f_{z}\right)$,

Whenever $f_{z}(x)=(z-x)^{n} U(z-x)$ and

$$
\begin{aligned}
& F_{Z_{1} \mid x_{1}, x_{2}}(f) \\
& =\frac{f_{z}\left(x_{1}\right)}{\left(x_{2}-x_{1}\right)^{n}}-\sum_{i=1}^{n} \frac{1}{(n-i) !\left(x_{2}-x_{1}\right)^{i}} \frac{\mathrm{d}^{n-i}}{\mathrm{~d} z^{n-i}} f_{z}\left(x_{2}\right) .
\end{aligned}
$$


Also we note that $U(z-x)=\frac{(-1)^{n}}{n !} \frac{\mathrm{d}^{n}}{\mathrm{~d} x^{n}} f_{z}(x)$. Thus

$$
\begin{aligned}
P\left(Z_{1} \leq z\right) & =\int_{\mathbb{R}} U(z-x) \mathrm{d} F_{Z_{1}}(x) \\
& =\int_{\mathbb{R}^{2}} F_{Z_{1} \mid x_{1}, x_{2}}(z) \prod_{i=1}^{2} F_{X_{i}}\left(\mathrm{~d} x_{i}\right),
\end{aligned}
$$

can be viewed as:

$$
\begin{aligned}
& \int_{\mathbb{R}} \frac{(-1)^{n}}{n !} \frac{\mathrm{d}^{n}}{\mathrm{~d} x^{n}} f_{z}(x) \mathrm{d} F_{Z_{1}}(x) \\
& =\int_{\mathbb{R}^{2}} F_{Z_{1} \mid x_{1}, x_{2}}\left(f_{z}\right) \prod_{i=1}^{2} F_{X_{i}}\left(\mathrm{~d} x_{i}\right) .
\end{aligned}
$$

Therefore by using (2.3) along with (2.4) and a standard argument in the integration theory, we obtain that

$$
\begin{aligned}
& \int_{\mathbb{R}} \frac{(-1)^{n}}{n !} \frac{\mathrm{d}^{n}}{\mathrm{~d} x^{n}} f(x) \mathrm{d} F_{Z_{1}}(x) \\
& =\int_{\mathbb{R}^{2}} F_{Z_{1} \mid x_{1}, x_{2}}(f) \prod_{i=1}^{2} F_{X_{i}}\left(\mathrm{~d} x_{i}\right) .
\end{aligned}
$$

For any infinitely differentiable functions $f$ for which the corresponding integrals are finite. Now (2.5) together with (2.2) lead us to

$$
\int_{\mathbb{R}} f(x) \mathrm{d}_{Z_{1}}{ }^{(n)}(x)=\int_{\mathbb{R}^{2}} F_{Z_{1} \mid x_{1}, x_{2}}(f) \prod_{i=1}^{2} F_{X_{i}}\left(\mathrm{~d} x_{i}\right) .
$$

For the above mentioned functions $f$, where $F_{Z_{1}}^{(n)}$ is the (n)-th distributional derivative of the distribution of $Z_{1}$.

Let us denote the Stieltjes transform of a distribution $H$ by

$$
S(H, z)=\int_{\mathbb{R}} \frac{1}{(z-x)} H(\mathrm{~d} x) .
$$

For every $z$ in the set of complex numbers $\mathbb{C}$ which does not belong to the support of $H$, i.e., $z \in \mathbb{C}(\operatorname{suppH})^{c}$.

The following lemma indicates how the Stieljes transform of $Z_{1}$ and $X_{1}, X_{2}$ are related.

Lemma 2.2. Let $Z_{1}$ be a random variables that satisfies (1.1). Suppose that the random variables $X_{1}$ and $X_{2}$ are independent and continuous with distribution functions $F_{X_{1}}$ and $F_{X_{2}}$ respectively. Then

$$
\begin{aligned}
& \frac{1}{n} S^{(n)}\left(F_{Z_{1}}, z\right)=-S\left(F_{X_{1}}, z\right) S^{(n-1)}\left(F_{X_{2}}, z\right), \\
& z \in \mathbb{C}(\operatorname{suppH})^{c} .
\end{aligned}
$$

Proof. It follows from (2.6) that

$$
S\left(F_{Z_{1}}{ }^{(n)}, z\right)=\int_{\mathbb{R}^{2}} F_{Z_{1} \mid x_{1}, x_{2}}\left(g_{z}\right) \prod_{i=1}^{2} F_{X_{i}}\left(\mathrm{~d} x_{i}\right) .
$$

And

$$
\frac{1}{n !} \frac{\mathrm{d}^{n}}{\mathrm{~d} z^{n}} S\left(F_{Z_{1}}, z\right)=\int_{\mathbb{R}^{2}} F_{Z_{1} \mid x_{1}, x_{2}}\left(g_{z}\right) \prod_{i=1}^{2} F_{X_{i}}\left(\mathrm{~d} x_{i}\right)
$$

for $g_{z}(x)=\frac{1}{z-x}$. Now, it follows that

$$
\begin{aligned}
& F_{Z_{1} \mid x_{1}, x_{2}}\left(g_{z}\right) \\
& =\frac{\frac{1}{z-x_{1}}}{\left(x_{2}-x_{1}\right)^{n}}-\sum_{i=1}^{n} \frac{1}{(n-i) !\left(x_{2}-x_{1}\right)^{i}} \frac{\mathrm{d}^{n-i}}{\mathrm{~d} z^{n-i}} \frac{1}{z-x_{2}} .
\end{aligned}
$$

And by using Lemma 2.1, we have

$$
F_{Z_{1} \mid x_{1}, x_{2}}\left(g_{z}\right)=\frac{(-1)^{n}}{\left(z-x_{1}\right)\left(z-x_{2}\right)^{n}} \text {. }
$$

Therefore,

$$
\frac{1}{n !} \frac{\mathrm{d}^{n}}{\mathrm{~d} z^{n}} S\left(F_{Z_{1}}, z\right)=\int_{\mathbb{R}^{2}} \frac{(-1)^{n}}{\left(z-x_{1}\right)\left(z-x_{2}\right)^{n}} \prod_{i=1}^{2} F_{X_{i}}\left(\mathrm{~d} x_{i}\right),
$$

and

$$
\begin{aligned}
& \frac{1}{n} S^{(n)}\left(F_{Z_{1}}, z\right)=-S\left(F_{X_{1}}, z\right) S^{(n-1)}\left(F_{X_{2}}, z\right), \\
& z \in \mathbb{C}(\operatorname{suppH})^{c} .
\end{aligned}
$$

This finishes the proof.

Note that Van Asch's lemma is the case of $n=1$ :

$$
-S^{\prime}\left(F_{Z_{1}}, z\right)=S\left(F_{X_{1}}, z\right) S\left(F_{X_{2}}, z\right) .
$$

We also note that the Stieltjes transform of Cauchy distribution, i.e., $S(F, z)=\frac{1}{z+c}$ satisfies (2.7).

\section{Characterizations}

Now, we apply Lemma 2.2 for some characterizations, when $X_{1}$ and $X_{2}$ are not identically distributed.

Theorem 3.1. Let $X_{1}$ and $X_{2}$ be independent random variables and $Z$ be a randomly weighted average given in (1.1).

For $n=2$ we have,

a) if $X_{1}$ has uniform distribution on $[-1,1]$, then $Z_{1}$ has semicircle distribution on $[-1,1]$ if and only if $X_{2}$ has Arcsin distribution on $[-1,1]$;

b) if $X_{1}$ has uniform distribution on $[-1,1]$, then $Z_{1}$ has power semicircle distribution on $[-1,1]$ if and only if $X_{2}$ has power semicircle distribution i.e.,

$$
f(z)=\frac{3\left(1-z^{2}\right)}{4},-1 \leq z \leq 1 .
$$

c) if $X_{1}$ has Beta $(1,1)$ distribution on $[0,1]$, then $Z_{1}$ has Beta $\left(\frac{3}{2}, \frac{3}{2}\right)$ distribution if and only if $X_{2}$ has Beta 
$\left(\frac{1}{2}, \frac{1}{2}\right)$ distribution;

d) if $X_{1}$ has uniform distribution on $[0,1]$, then $Z_{1}$ has Beta $(2,2)$ distribution if and only if $X_{2}$ has Beta $(2,2)$ distribution.

Proof. 1) For the "if" part we note that the random variable $X_{1}$ has uniform distribution and $X_{2}$ has Arcsin distribution on $[-1,1]$; then

$$
S\left(F_{X_{1}}, z\right)=\frac{1}{2}(\ln |z+1|-\ln |z-1|) .
$$

And $S\left(F_{X_{2}}, z\right)=\frac{1}{\sqrt{z^{2}-1}}$.

From Lemma 2.2 and substituting the corresponding Stieltjes transforms of distributions, we get

$$
S^{\prime \prime}\left(F_{Z_{1}}, z\right)=\frac{2}{\left(z^{2}-1\right)^{\frac{3}{2}}} \text {. }
$$

The solution $S\left(F_{Z_{1}}, z\right)=2\left(z-\sqrt{z^{2}-1}\right)$.

Which is the Stieltjes transform of the semicircle distribution on $[-1,1]$.

For the "only if" part we assume that the random variable $Z_{1}$ has semicircle distribution. Then it follows from Lemma 2.2 that

$$
S\left(F_{X_{2}}, z\right) \frac{1}{1-z^{2}}=\frac{-1}{\left(z^{2}-1\right)^{\frac{3}{2}}} .
$$

The proof is completed.

2) By an argument similar to that given in 1) and solving the following differential equations,

$$
\begin{aligned}
& S^{\prime \prime}\left(F_{Z_{1}}, z\right) \\
& =\frac{-2}{z(z-1)}\left(6\left(z^{2}-z\right)(\ln |z|-\ln |z-1|)-6 z+3\right) .
\end{aligned}
$$

(for the "if" part), and

$$
\mathrm{S}\left(F_{X_{2}}, z\right)=6\left(z^{2}-z\right)(\ln |z|-\ln |z-1|)+6 z-3 .
$$

(for the "only if" part).

The proof can be completed.

3) By Lemma (2.2), we have

$$
-\frac{1}{2} S^{\prime \prime}\left(F_{Z}, z\right)=\frac{-1}{z(z-1) \sqrt{z(z-1)}},
$$

(for the "if" part), and

$$
-\frac{1}{z(z-1)} S\left(F_{X_{2}}, z\right)=\frac{-1}{z(z-1) \sqrt{z(z-1)}},
$$

(for the "only if" part).

The proof can be completed by solving the above dif- ferential equations.

4) By Lemma (2.2), we have

$$
\begin{aligned}
& S^{\prime \prime}\left(F_{Z_{1}}, z\right) \\
& =\frac{-2}{z(z-1)}\left(6\left(z^{2}-z\right)(\ln |z|-\ln |z-1|)-6 z+3\right)
\end{aligned}
$$

(for the "if" part), and

$$
\mathrm{S}\left(F_{X_{2}}, z\right)=6\left(z^{2}-z\right)(\ln |z|-\ln |z-1|)+6 z-3
$$

(for the "only if" part).

Solving the differential equations, can complete the proof.

\section{TSP Random Variables}

In Section 3, we used a powerful method, based on the use of Stieltjes transforms, to obtain the distribution of $z_{1}$ given in (1.1). It seems that one can not use that method to find distribution of $z_{2}$ given in (1.2). So we employ a direct method to find the distribution of $z_{2}$. Let us follow Lemma 4.1 to find a simple method to get the distribution of $z_{2}$ following [2] and the work of them leads us to the following lemma.

Lemma 4.1. Suppose $W$ has a power distribution with parameter $n, n \geq 1, n$ is an integer, and let $y_{1}=\min \left(X_{1}, X_{2}\right), \quad y_{2}=\max \left(X_{1}, X_{2}\right)$ where $X_{1}, \cdots, X_{n}$ independent random variables are. Let

$$
X=Y_{1}+W\left(Y_{2}-Y_{1}\right) .
$$

Then

1) $X$ is a TSP random variable.

2) $X$ can be equivalently defined by

$$
X=\frac{1}{2}\left(X_{1}+X_{2}\right)+\left(W-\frac{1}{2}\right)\left|X_{1}-X_{2}\right| .
$$

Proof. 1)

$$
\begin{aligned}
F_{X \mid x_{1}, x_{2}}(z) & =\left(P Y_{1}+W\left(Y_{2}-Y_{1}\right) \leq z \mid X_{1}=x_{1}, X_{2}=x_{2}\right) \\
& =P\left(y_{1}+W\left(y_{2}-y_{1}\right) \leq z\right)=\left(\frac{z-y_{1}}{y_{2}-y_{1}}\right)^{n} .
\end{aligned}
$$

Proof. 2)

$$
\begin{gathered}
X \mid X_{1}=x_{1}, X_{2}=x_{2} \sim U\left[\min \left(x_{1}, x_{2}\right), \max \left(x_{1}, x_{2}\right)\right], \\
W=\frac{X-\min \left(x_{1}, x_{2}\right)}{\max \left(x_{1}, x_{2}\right)-\min \left(x_{1}, x_{2}\right)} \sim U[0,1],
\end{gathered}
$$

and also

$$
\begin{aligned}
& \min \left(x_{1}, x_{2}\right)=\frac{x_{1}+x_{2}-\left|x_{1}-x_{2}\right|}{2}, \\
& \max \left(x_{1}, x_{2}\right)=\frac{x_{1}+x_{2}+\left|x_{1}-x_{2}\right|}{2} .
\end{aligned}
$$


then

$$
W=\frac{X-\frac{x_{1}+x_{2}}{2}-\frac{\left|x_{1}-x_{2}\right|}{2}}{\left|x_{1}-x_{2}\right|},
$$

so

$$
X=\frac{1}{2}\left(x_{1}+x_{2}\right)+\left(W-\frac{1}{2}\right)\left|x_{1}-x_{2}\right|
$$

\subsection{Moments of TSP Random Variables}

The following theorem provides equivalent conditions For $\mu_{X}^{\prime}=E z_{2}^{k}$.

Theorem 4.1.1. Suppose that $z_{2}$ is a TSP random variable satisfying (1.2). If $X_{1}$ and $X_{2}$ are random variables and $E\left|X_{i}\right|^{k}=\infty, i=1,2$ for all integers $k$ then

1) $E z_{2}^{k}=n \frac{\Gamma(k+1)}{\Gamma(n+k+1)} \sum_{i=0}^{k} \frac{\Gamma(k+i-n)}{\Gamma(k-i+1)} E\left(y_{1}^{i} y_{2}^{k-i}\right)$.

2) $E z_{2}^{k}=\left(\begin{array}{c}k \\ i\end{array}\right)\left(\frac{1}{2}\right)^{k-i} E\left(W-\frac{1}{2}\right)^{i} E\left(X_{1}+X_{2}\right)^{k-i}\left|X_{1}+X_{2}\right|^{i}$.

3) $E z_{2}^{k}=\left(\begin{array}{l}k \\ i\end{array}\right)\left(\frac{n}{n+i}\right) E\left(y_{1}^{k-i}\left(y_{2}-y_{1}\right)^{i}\right)$.

Proof. 1) By using Lemma 2.1, we obtain that

$$
\begin{aligned}
& =\sum_{i=0}^{k}\left(\begin{array}{l}
k \\
i
\end{array}\right) E\left(W^{k-i}(1-W)^{i}\right) E\left(Y_{2}^{k-i} Y_{1}^{i}\right) \\
& =\sum_{i=0}^{k}\left(\begin{array}{l}
k \\
i
\end{array}\right) E\left(W^{k-i}(1-W)^{i}\right) E\left(Y_{2}^{k-i} Y_{1}^{i}\right) \\
& =n \frac{\Gamma(k+1)}{\Gamma(n+k+1)} \sum_{i=0}^{k} \frac{\Gamma(k-i+n)}{\Gamma(k-i+1)} E\left(y_{1}^{i} y_{2}^{k-i}\right) .
\end{aligned}
$$

Proof. 2) This can be easily proved by Lemma 4.12).

Proof. 3)

$$
\begin{aligned}
E z_{2}^{k} & =\left(Y_{1}+W\left(Y_{2}-Y_{1}\right)\right)^{k} \\
& =E \sum_{i=0}^{k}\left(\begin{array}{c}
k \\
i
\end{array}\right)\left(W^{i} y_{1}^{k-i}\left(y_{2}-y_{1}\right)^{i}\right) \\
& =\sum_{i=0}^{k}\left(\begin{array}{c}
k \\
i
\end{array}\right) E(W)^{i} E\left(y_{1}^{k-i}\left(y_{2}-y_{1}\right)^{i}\right) \\
E z_{2}^{k} & =\left(\begin{array}{c}
k \\
i
\end{array}\right)\left(\frac{n}{n+i}\right) E\left(y_{1}^{k-i}\left(y_{2}-y_{1}\right)^{i}\right) .
\end{aligned}
$$

Let us consider expectation and variance of $z_{2}$. First, we suppose that $E Y_{1}=\mu_{1}, E Y_{2}=\mu_{2}, \operatorname{Var} Y_{1}=\sigma_{1}^{2}, \operatorname{Var} Y_{2}=$ $\sigma_{2}^{2}$, and $\operatorname{Cov}\left(Y_{1}, Y_{2}\right)=\sigma_{12}$. Then

$$
E Z_{2}=\frac{\mu_{1}+n \mu_{2}}{n+1},
$$

and also, if $E X_{1}=E X_{2}=0$ then

$$
E\left(Z_{2}\right)=E Y_{1}+\frac{n}{n+1}\left(E Y_{2}-E Y_{1}\right) .
$$

By $X_{1}+X_{2}=Y_{1}+Y_{2}$. We have

$$
E\left(Z_{2}\right)=E\left(Y_{1}\right)+\frac{n}{n+1}\left(-2 E Y_{1}\right)=\frac{1-n}{1+n} E Y_{1} .
$$

It can easily follow from (4.2) that the Arcsin result of Van Asch [1] is only true for $n=1$, about the variance, we have

$$
\begin{aligned}
& \operatorname{VarZ} Z_{2} \\
& =\frac{n\left(\mu_{1}-\mu_{2}\right)^{2}+n(n+1)^{2} \sigma_{2}^{2}+2(n+1)\left(\sigma_{1}^{2}+n \sigma_{12}\right)}{(n+1)^{2}(n+2)} .
\end{aligned}
$$

Following the computation of expectation and variance, we evaluate them for some well-known distributions. If $X_{1}$ and $X_{2}$ have standard normal distributions, then from Theorem 3.1.12) and the fact that $X_{1}-X_{2}$ and $X_{1}+X_{2}$ are independent, it follows that their first, second and third order moments are equal, respectively, to

$$
\begin{gathered}
E Z_{2}=\frac{1}{\sqrt{\pi}}\left(\frac{n-1}{n+1}\right), \\
E Z_{2}^{2}=\left(\frac{n^{2}+n+2}{(n+1)(n+2)}\right),
\end{gathered}
$$

and

$$
E Z_{2}^{3}=\frac{1}{2 \sqrt{\pi}}\left(\frac{5 n^{3}+12 n^{2}+13 n-30}{(n+3)(n+1)(n+2)}\right) .
$$

Also, in case $X_{1}$ and $X_{2}$ have uniform distributions, Theorem 4.1.1 2) implies that,

$$
\begin{gathered}
E z_{2}^{k}=n \frac{\Gamma(k+1)}{\Gamma(n+k+1)} \sum_{i=0}^{k} \frac{\Gamma(k-i+n)}{\Gamma(k-i+1)} \frac{2}{(k+2)(i+1)} . \\
E Z_{2}=\frac{2 n+1}{3(n+1)},
\end{gathered}
$$

and

$$
\operatorname{Var}_{2}=\frac{1}{18} \frac{n^{3}+3 n^{2}+6 n+2}{(n+1)^{2}(n+1)} .
$$

Theorem 4.1.2. Suppose that $z_{2}$ is a TSP random variable satisfying (4.1), then

1) $z_{2}$ is location invariant;

2) If $X_{1}$ and $X_{2}$ have symmetric distribution around $\mu$, then $z_{2}$ has symmetric distribution around $\mu$, only when $n=1$.

Proof.

1) Is immediate.

2) We can assume without loss of generality that $\mu=0$ If $Z_{2}$ has a symmetric distribution around zero, then 


$$
Y_{1}+W\left(Y_{2}-Y_{1}\right) \stackrel{d}{=}-\left[Y_{1}+W\left(Y_{2}-Y_{1}\right)\right] .
$$

We note that

$$
Y_{1}+W\left(Y_{2}-Y_{1}\right) \stackrel{d}{=}\left[-Y_{1}+W\left(-Y_{2}-\left(-Y_{1}\right)\right)\right] .
$$

Since $-\min \left(X_{1}, X_{2}\right)=\max \left(-X_{1},-X_{2}\right), \quad X_{1} \stackrel{d}{=}-X_{1}$, And $X_{2} \stackrel{d}{=}-X_{2}$, we have

$$
Y_{1}+W\left(Y_{2}-Y_{1} \stackrel{d}{=} Y_{2}+W\left(Y_{1}-Y_{2}\right) .\right.
$$

By equating the conditional distributions given at $X_{1}=x_{1}$ and $X_{2}=x_{2}$ in (3.3), we conclude that $n=1 \mathrm{It}$ can also easily follow from Theorem (4.1.1) that the Cauchy result of Van Asch [1] is true only for $n=1$.

\subsection{Distributions of TSP Random Variables}

In this subsection, we investigate computing distributions by the direct method. We will give two examples of derivation based on (4.1). This method may be complicated in some cases, but we have chosen some easy to find examples. We use randomly weighted average on order statistics to find the distribution of $z_{2}$. Gauss hyper geometric function $F(a, b, c ; z)$ which is a well-known special function that we used in this way.

Example 4.2.1. Let $X_{1}, X_{2}$ and $\mathrm{W}$ be independent random variables such that $X_{1}$ and $X_{2}$ are uniformly distributed over $[0,1]$, and $\mathrm{W}$ has a power function distribution with parameter. We find the value $f_{z_{2}}(z ; n)$ by means of $f_{Z_{2} \mid W(z \mid w)}$; therefore

$$
f_{Z_{2} \mid W(z \mid w)}= \begin{cases}\frac{2 z}{w} & 0<z<w, \\ \frac{2(1-z)}{1-w} & w<z<1 .\end{cases}
$$

By using the distribution of $W$, the density function $f_{Z_{2}}(z ; n)$, can be expressed in terms of the Gauss hyper geometric function $F(a, b, c ; z)$ which is a well-known special function. Indeed according to Euler's formula, the Gauss hyper geometric function assumes the integral representation

$$
\begin{aligned}
& F(a, b, c ; z) \\
& =\frac{\Gamma(c)}{\Gamma(b) \Gamma(c-b)} \int_{0}^{1} t^{b-1}(1-t)^{c-b-1}(1-t z)^{-a} \mathrm{~d} t,
\end{aligned}
$$

where $a, b, c$ are parameters subject to $-\infty<a<\infty$, $c>b>0$, whenever they are real and $z$ is the variable.

$$
\begin{aligned}
& f_{Z_{2}}(z ; n) \\
& =\frac{2 n z}{n-1}\left(1-z^{n-1}\right)+2(1-z) z^{n} F(1, n, n+1, z),
\end{aligned}
$$

where $n>0$ and $n \neq 1$. There are some important functions as a Gauss hyper geometric function.

$$
\begin{gathered}
\log (1+z)=z F(1,1 ; 2 ;-Z) . \\
\mathrm{e}^{z}=\lim _{b \rightarrow \infty} F\left(a, b ; b ; \frac{z}{a}\right) . \\
(1-z)^{-a}=F(a, 1 ; 1 ; z) .
\end{gathered}
$$

When $n=1$ similar calculations lead to the following distribution

$$
f_{Z_{2}}(z)=-2(1-z) \log (1-z)-2 z \log (z), 0<z<1 .
$$

When $n$ is an integer, we obtain the following distribution.

$$
\begin{aligned}
f_{Z_{2}}^{*}(z, n)= & 2 \frac{n}{n-1} z\left(1-z^{n-1}\right) \\
& -2 n z(1-z) \sum_{i=0}^{n-1}\left(\begin{array}{c}
n-1 \\
i
\end{array}\right) \frac{(-1)^{i}}{i}\left((1-z)^{i}-1\right),
\end{aligned}
$$

$0<z<1$.

The probability density function $f_{Z_{2}}(z)$ was introduced by Johnson and Kotz [3], for the first time, under the title "uniformly randomly modified tine". So $f_{Z_{2}}(z ; n)$ can be seen as an extension of the above mentioned distribution. We note that, from (4.1) and a simple Monte Carlo procedure using only simulated uniform variables, one can to simulate the distribution (4.5).

Theorem 4.2.2. Let $z_{2}$ be a undirected triangular random variable that satisfies (1.2). Suppose that the random variables $X_{1}$ and $X_{2}$ are independent and continuous with the distribution Functions $F_{X_{1}}$ and $F_{X_{2}}$, respectively. Then

$$
-\frac{1}{2} S^{\prime \prime \prime}\left(F_{Z}, z\right)=S^{\prime}\left(F_{X_{1}}, z\right) S^{\prime}\left(F_{X_{2}}, z\right)+2 S\left(F_{X_{1}}, F_{X_{2}}, z\right),
$$

where

$$
\begin{aligned}
& S\left(F_{X_{1}}, F_{X_{2}}, z\right) \\
& =\int_{\mathbb{R}^{2}} \frac{1}{\left(z-x_{1}\right)\left(z-x_{2}\right)\left(x_{2}-x_{1}\right)^{2}} \prod_{i=1}^{2} F_{X_{i}}\left(\mathrm{~d} x_{i}\right) .
\end{aligned}
$$

Proof. By using an argument similar to that given in section 3 , we can conclude that

$$
\int_{\mathbb{R}} f(x) \mathrm{d}_{Z_{2}}^{(2)}(x)=\int_{\mathbb{R}^{2}} F_{Z_{2} \mid x_{1}, x_{2}}(f) \prod_{i=1}^{2} F_{X_{i}}\left(\mathrm{~d} x_{i}\right) .
$$

So,

$$
-\frac{1}{2} S^{\prime \prime \prime}\left(F_{Z_{2}}, z\right)=\int_{\mathbb{R}^{2}} F_{Z_{2} \mid x_{1}, x_{2}}\left(g_{z}\right) \prod_{i=1}^{2} F_{X_{i}}\left(\mathrm{~d} x_{i}\right),
$$

For $g_{z}(x)=\frac{1}{(z-x)^{2}}$. From 


$$
F_{Z_{2} \mid x_{1}, x_{2}}\left(g_{z}\right)=\frac{\frac{1}{\left(z-x_{1}\right)^{2}}}{\left(x_{2}-x_{1}\right)^{2}}+\frac{1}{\left(z-x_{2}\right)^{2}} .
$$

And by using partial fractional rule, we have

$$
\begin{aligned}
& F_{z_{2} \mid x_{1}, x_{2}}\left(g_{z}\right) \\
& =\frac{1}{\left(z-x_{1}\right)^{2}\left(z-x_{2}\right)^{2}}+\frac{2}{\left(x_{2}-x_{1}\right)^{2}} \frac{1}{\left(z-x_{1}\right)\left(z-x_{2}\right)} .
\end{aligned}
$$

Therefore,

$$
\begin{aligned}
& -\frac{1}{2} S^{\prime \prime \prime}\left(F_{Z_{2}}, z\right)=\int_{\mathbb{R}^{2}} \frac{1}{\left(z-x_{1}\right)^{2}\left(z-x_{2}\right)^{2}} \\
& +\frac{2}{\left(x_{2}-x_{1}\right)^{2}} \frac{1}{\left(z-x_{1}\right)\left(z-x_{2}\right)} \prod_{i=1}^{2} F_{X_{i}}\left(\mathrm{~d} x_{i}\right) .
\end{aligned}
$$

And

$-\frac{1}{2} S^{\prime \prime \prime}\left(F_{Z}, z\right)=S^{\prime}\left(F_{X_{1}}, z\right) S^{\prime}\left(F_{X_{2}}, z\right)+2 S\left(F_{X_{1}}, F_{X_{2}}, z\right)$.

This finishes the proof.

It is worth mentioning that the present method yields other extensions too; the following is such an example.

Example 4.2.3. Suppose that $X_{1}, X_{2} W$ are independent random variables. If $X_{1}$ and $X_{2}$ have Uniform distributions on $[0,1]$ and $W$ has Beta $(2,2)$ distribution, then $z_{2}$ has the same distribution as $W$.

If the product moments of order statistics are known, those of $W$ can be derived from that of $z_{2}$. By using Theorem 4.1.1 1). Then the distribution of $W$ is characterized by that of $z_{2}$.

By an argument similar to the one given in example 4.2.1, when $W$ has a Beta distribution with Parameters $n$ and $m$, we find the distribution $f_{Z_{2}}(z ; n, m)$ as

$$
\begin{aligned}
& \frac{B(n-1, m)}{B(n, m)} 2 z\left(1-I_{z}(n-1, m)\right) \\
& +\frac{B(n, m-1)}{B(n, m)} 2(1-z) I_{z}(n, m-1), \\
& 0<z<1
\end{aligned}
$$

where $I_{z}(a, b)$ is incomplete Beta function:

$$
I_{x}(a, b)=\frac{1}{B(a, b)} \int_{0}^{x} t^{a-1}(1-t)^{b-1} \mathrm{~d} t,(a, b>0) .
$$

\section{Conclusion}

We have described how directed methods could be used for obtaining the distributions, Characterizations and properties of the random mixture of variables defined in (1.1). The TSP random variable when $X_{1}$ and $X_{2}$ have uniform distributions, led us to a new family of distribution which can be regarded as some generalization of "uniformly randomly modified tine". The proposed model in the direct method can easily lead to distribution generalizations, though this is not possible for the Stieltjes method, but here the characteristics can be easily computed.

\section{Acknowledgements}

The author is deeply grateful to the anonymous referee for reading the original manuscript very carefully and for making valuable suggestions.

\section{REFERENCES}

[1] W. Van Asch, "A Random Variable Uniformly Distributed between Two Independent Random Variables," Sankhaya, Vol. 49, No. 2, 1987, pp. 207-211. doi:10.1080/00031305.1990.10475730

[2] A. R. Soltani and H. Homei, "Weighted Averages with Random Proportions That Are Jointly Uniformly Distributed over the Unit Simplex," Statistics \& Probability Letters, Vol. 79, No. 9, 2009, pp. 1215-1218. doi:10.1016/j.spl.2009.01.009

[3] N. L. Johnson and S. Kotz, "Randomly Weighted Averages," The American Statistician, Vol. 44, No. 3, 1990, pp. 245-249. doi:10.2307/2685351

[4] A. I. Zayed, "Handbook of Function and Generalized Function Transformations," CRC Press, London, 1996.

[5] A. R. Soltani and H. Homei, "A Generalization for TwoSided Power Distributions and Adjusted Method of Moments," Statistics, Vol. 43, No. 6, 2009, pp. 611-620. doi: $10.1080 / 02331880802689506$ 